\title{
新型蒎烷基吡唑酰胺类衍生物的合成及其生物活性研究
}

\author{
苪 坚 $a$ 张 齐 $a$ 王 欣 ${ }^{b}$ 徐 徐 ${ }^{a, c, d}$ 徐海军 ${ }^{a}$ \\ 饶卫东 ${ }^{a}$ 王石发 $*, a, c, d$ \\ ( ${ }^{a}$ 南京林业大学化学工程学院 南京 210037) \\ ( ${ }^{b}$ 南京农业大学植物保护学院 南京 210095) \\ ( ${ }^{c}$ 江苏省生物质绿色燃料与化学品重点实验室 南京 210037) \\ ( ${ }^{d}$ 广西林产化学与工程重点实验室 南宁 530008)
}

\begin{abstract}
摘要 以诺蒎酮为原料, 合成了新型蒎烷基吡唑酰胺类衍生物, 并对其生物活性进行了研究. 诺蒎酮与芳香醛进行缩 合反应，得到中间体 3-芳亚甲基诺蒎酮 $\mathbf{2 a} \sim 2 \mathrm{~g}, \mathbf{2 a} \sim 2 \mathrm{~g}$ 与水合肼经环化、脱氢芳构化反应，得到新型蒎烷基吡唑类化 合物 3a $\sim 3 \mathbf{g}$; 以 $N, N$-二甲基甲酰胺为溶剂, 3a $\sim 3 \mathbf{g}$ 与 2-氯乙酰胺在碱催化作用下，合成了 7 种吡唑酰胺类化合物 $4 \mathbf{a} \sim$ 4g. 其结构经 ${ }^{1} \mathrm{H} N M R,{ }^{13} \mathrm{C} N M R, I R$, 和 HRMS 表征. 通过 X 射线衍射分析测定了化合物 2-(6',6'-二甲基-3'-(4'-甲氧基 苯基)-4', 5',6', 7'-四氢-5',7'-桥亚甲基-吲唑-1'-基)乙酰胺(4d)的晶体结构. 探讨了化合物 $\mathbf{3 a} \sim \mathbf{3 g}$ 和 $\mathbf{4 a} \sim \mathbf{4 g}$ 的抑菌活性和对 蚜虫的杀虫活性. 实验结果表明, 2-(6',6'-二甲基-3'-(4'-氟苯基)-4', $5^{\prime}, 6^{\prime}, 7^{\prime}$-四氢-5',7'-桥亚甲基-吲唑-1'-基)乙酰胺(4g)既具 有较好的抑菌效果, 同时对紫薇蚜虫也表现出较好的杀虫活性.
\end{abstract}

关键词 诺蒎酮; 蒎烷基吡唑衍生物; 抑菌活性; 杀虫活性

\section{Synthesis and Biological Activity of Novel Pinanyl Pyrazole Acetamide Derivatives}

\author{
Rui, $\operatorname{Jian}^{a}$ \\ Zhang, $\mathrm{Qi}^{a}$ \\ Wang, Xin ${ }^{b}$ \\ $\mathrm{Xu}, \mathrm{Xu}^{a, c, d}$ \\ $\mathrm{Xu}$, Haijun $^{a}$ \\ Rao, Weidong ${ }^{a}$ \\ Wang, Shifa*,a,c,d \\ ( ${ }^{a}$ College of Chemical Engineering, Nanjing Forestry University, Nanjing 210037) \\ ( ${ }^{b}$ College of Plant Protection, Nanjing Agricultural University, Nanjing 210095) \\ ( ${ }^{c}$ Jiangsu Key Laboratory of Biomass-based Green Fuels and Chemicals, Nanjing 210037) \\ ( ${ }^{d}$ Guangxi Key Laboratory of Chemistry and Engineering of Forest Products, Nanning 530008)
}

\begin{abstract}
Novel pinanyl pyrazole derivatives were synthesized from nopinone and their bioactivities were also examined in this paper. The intermediate compounds 3-arylidenenopinones $\mathbf{2 a} \sim \mathbf{2} \mathbf{g}$ were synthesized by condensation of aromatic aldehydes and nopinone, and pinanyl pyrazole derivatives $\mathbf{3 a} \sim \mathbf{3 g}$ were subsequently obtained by cyclization and dehydroaromatization of compounds $\mathbf{2} \mathbf{a} \sim \mathbf{2 g}$ and hydrazine hydrate in the circumstance of $N, N$-dimethylformamide (DMT). The target compound pinanyl pyrazole acetamide derivatives $\mathbf{4 a} \sim \mathbf{4 g}$ were synthesized by alkylation using 2-chloroacetamide as the alkylating agent under the condition of base catalysis. The structures of synthesized compounds were characterized by ${ }^{1} \mathrm{H} N M R,{ }^{13} \mathrm{C}$ NMR, IR, HRMS, and X-ray diffraction analysis. Biological activities evaluation of compounds $3 \mathbf{a} \sim \mathbf{3 g}$ and $\mathbf{4 a} \sim \mathbf{4 g}$ were performed including antibacterial and insecticidal activities on Tinocallis kahawaluokalani. Test results showed that compound $\mathbf{4 g}$ was a more potent bactericide and fungicide than others because of its good activities against bacteria and fungi; furthermore, compound $\mathbf{4 g}$ had shown the better insecticidal activity against Tinocallis kahawaluokalani than the others.
\end{abstract}

Keywords nopinone; pinanyl pyrazole derivatives; bacteriostatic activity; insecticidal activity

\footnotetext{
* Corresponding author. E-mail: wsfyyq@njfu.com.cn

Received June 24, 2016; revised August 4, 2016; published online September 1, 2016.

Project supported by the Natural Science Foundation of the Jiangsu Higher Education Institutions of China (No. 14KJ220001), the National Natural Science Foundation of China (No. 31470529), and the Open Funding of Guangxi Key Laboratory of Chemistry and Engineering of Forest Products (No. GXFC15-02). 江苏省高校自然科学研究重大项目(No. 14KJ220001)、国家自然科学基金(No. 31470529)和广西林产化学与工程重点实验室开放基金(No. JSBEM2014010)资助项目.
} 
吡唑具有两个杂原子形成的五元含氮环和共轭体 系, 这种结构使吡唑环表现出许多独特的生物活性, 吡 唑类化合物在医药、农药等领域有着广泛的应用 ${ }^{[1 \sim 4]}$, 如抗虫、杀菌和除草活性等 ${ }^{[5 \sim 10]}$. 在吡唑类化合物中, 吡唑酰胺类化合物由于具有低毒、高效等优良的杀 虫 ${ }^{[10 ~ 13]}$ 活性而倍受人们的关注, 其分子设计、合成与生 物活性研究是当今农药创制的一个热点. 目前商品化的 吡坐类杀虫剂氟虫腈、吡螨胺、敌蝇威 ${ }^{[14]}$ 等. 氟虫腈是 一个极具代表性的芳香基吡唑类杀虫剂, 但氟虫腈 ${ }^{[15]}$ 对蜜蜂和水生生物毒性很大、对环境不友好制约了该药 的广泛使用. 另一方面, 植物源农药 ${ }^{[16]}$ 以其对害虫的作 用机制独特、不容易产生抗药性以及对天敌及人、畜低 毒, 无残留污染等优点, 被称为绿色农药. 如除虫菊素 是拟除虫菊酯类杀虫剂的先导化合物等. 本文利用具有 生物活性的天然产物萜烯类 ${ }^{[17-21]}$ 物质 $\beta$-蒎烯为原料, 采用活性拼接的方法, 将苯基和吡唑环引入蒎烷基中, 以诺蒎酮为先导化合物, 经与芳香醛缩合后得到 3-芳亚 甲基诺蒎酮, 再与水合肼环化之后进行脱氢芳构化反 应，合成了 7 个新型蒎烷基吡唑类化合物 $\mathbf{3 a} \sim \mathbf{3 g}$. 化合 物 3a $3 \mathbf{g}$ 与 2-氯乙酰胺在碱催化作用下, 合成了 7 种 吡唑酰胺类化合物 $\mathbf{4 a} \sim \mathbf{4 g}$. 化合物结构经 ${ }^{1} \mathrm{H} \mathrm{NMR,}{ }^{13} \mathrm{C}$ NMR，IR，MS 和 HRMS 确证. 初步生物活性测试表明, 部分化合物具有较好的抑菌活性和杀虫活性. 目标化合 物的合成路线见图 1 .

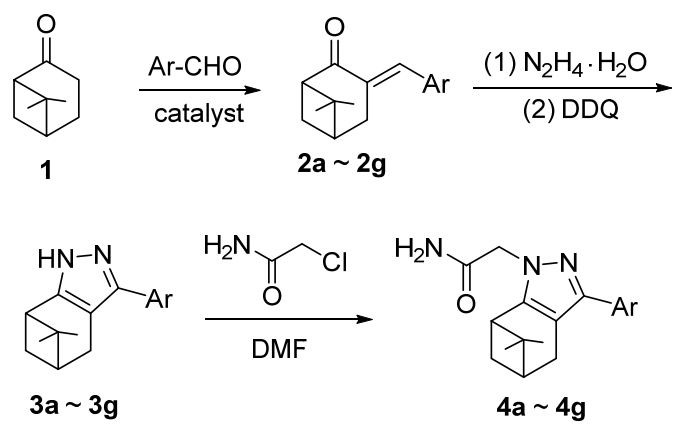

Ar: $p-\mathrm{CH}_{3} \mathrm{C}_{6} \mathrm{H}_{4}$ (4a), $p-\mathrm{ClC}_{6} \mathrm{H}_{4}$ (4b), $p-\mathrm{BrC}_{6} \mathrm{H}_{4}$ (4c), $p-\mathrm{CH}_{3} \mathrm{OC}_{6} \mathrm{H}_{4}$ (4d), $o-\mathrm{ClC}_{6} \mathrm{H}_{4}(\mathbf{4 e}), p-\mathrm{NO}_{2} \mathrm{C}_{6} \mathrm{H}_{4}(\mathbf{4 f}), p-\mathrm{FC}_{6} \mathrm{H}_{4}(\mathbf{4 g})$

图式 1 目标化合物的合成

Scheme 1 Synthesis of target compounds

\section{1 结果与讨论}

\section{1 目标化合物的合成讨论}

目标化合物的合成路线见 Scheme 1, 所用原料诺蒎 酮为实验室内自制, 采用高锰酸钾氧化 $\beta$-蒎烯制得 ${ }^{[22]}$. 在制备原料诺蒎酮 $\mathbf{1}$ 的过程中, 利用化合物沸点不同采 用蒸馏方法得到高纯度诺蒎酮; 在制备中间体 3-芳亚甲 基诺蒎酮 $2 \mathrm{a} \sim 2 \mathrm{~g}$ 的过程中, 采用重结晶的方法得到高
纯度中间体; 在合成 $3 \mathbf{a} \sim \mathbf{3 g}$ 过程中，未分离纯化与水合 肼反应后得到的吡唑啉中间体，直接脱氢芳构化制得蒎 烷基吡唑类化合物 $\mathbf{3 a} \sim \mathbf{3 g}$, 这是由于中间体吡唑啉不 稳定, 提纯过程中易分解. 在脱氢芳构化反应中, 相对 于 $\mathrm{KMnO}_{4}, \mathrm{PCC}, \mathrm{H}_{2} \mathrm{O}_{2}$ 等氧化剂而言, 选用 2,3-二 氯-5,6-二氰对苯醌(DDQ)作氧化剂, 反应转化率在 $90 \%$ 以上，反应选择性在 $80 \%$ 左右. 为了更好地去除杂质， 采用硅胶色谱柱纯化粗产品, 再经甲醇或乙腈重结晶得 到目标化合物 3a 3g. 在制备 $4 \mathrm{a} \sim \mathbf{4 g}$ 的过程中, 使用 展开剂石油醚：乙酸乙酯 $=1: 1$ 进行柱色谱分离得到 高纯度新型吡唑酰胺类化合物 $\mathbf{4 a} \sim \mathbf{4 g}$.

\section{2 结构表征}

分析化合物的 ${ }^{1} \mathrm{H} N \mathrm{NR}$ 数据发现, 在所合成中间体 $\mathbf{2 a} \sim \mathbf{2 g}$ 中, 在 $\delta 7.01 \sim 8.29$ 之间的多重峰是芳亚甲基上 氢的吸收信号, 氢原子个数与预期一致. 中间体 $\mathbf{2 a} \sim \mathbf{2 g}$ 经缩合、环化和脱氢芳构化反应后, 由不饱和酮转化成 具有芳香结构的吡唑环, 此时吡坐环上 NH 的质子信号 在 $\delta 12.40$ 附近, 而且是一个宽峰. 化合物 $\mathbf{4 a} \sim \mathbf{4 g}$ 酰胺 基团 $\mathrm{NH}_{2}$ 的质子信号在 $\delta 4.70$ 附近, 在 $\delta 12.40$ 附近没 有出现任何信号. 在 ${ }^{13} \mathrm{C} \mathrm{NMR}$ 中, 饱和碳的化学位移均 在高场区, 出现在 $\delta \quad 21.19 \sim 41.24$ 之间, 苯环和吡唑环 上不饱和碳的化学位移在 $\delta 106.34 \sim 162.74$ 范围内, 中 间体 $2 \mathbf{a} \sim 2 \mathbf{g}$ 的羰基碳化学位移在 $\delta 202.00$ 附近, 中间体 $3 \mathbf{a} \sim 3 \mathbf{g}$ 吡唑环上 2 位上的碳出现在最低场, 化学位移在 $\delta 158.00$ 附近, 吡唑酰胺 $\mathbf{4 a} \sim \mathbf{4 g}$ 的羰基碳化学位移在 $\delta$ 168.00 附近. 红外光谱中, 在 $3150 \mathrm{~cm}^{-1}$ 附近区域出现 $\mathrm{N}-\mathrm{H}$ 键的伸缩振动吸收峰; 3035 3100 $\mathrm{cm}^{-1}$ 之间出现 芳基 C-H 伸缩振动的特征吸收峰; 2866 2980 $\mathrm{cm}^{-1}$ 之 间是庚基上甲基及亚甲基的 $\mathrm{C}-\mathrm{H}$ 伸缩振动吸收峰; 在 $1439 \sim 1598 \mathrm{~cm}^{-1}$ 区域有苯环骨架的伸缩振动吸收峰; 在 $815 \sim 855 \mathrm{~cm}^{-1}$ 区域的吸收峰为对位二取代苯环上 $\mathrm{C}-\mathrm{H}$ 的面外弯曲振动. 在 $\mathrm{EI} / \mathrm{MS}$ 谱图中, 目标化合物 的分子离子峰与理论值也均吻合. 目标产物的 HRMS $[\mathrm{M}+\mathrm{H}]^{+}$实测值和计算值误差基本在 $0.5 \%$ 以内.

为了对该类系列化合物分子结构作进一步的验证, 选取化合物 4d 进行了 $X$ 射线单晶衍射分析. 单晶 $X$ 衍 射测试表明, 化合物 $4 \mathbf{d}$ 的晶体属正交晶系(orthorhombic), $P_{21} / c$ 空间群. 化合物的晶体结构见图 1 所示.

\section{3 抑菌活性测试}

采用微量二倍稀释法测定了目标化合物对 3 种真 菌：热带假丝酵母(G. tropicalis)、黑曲霉(A. niger)、白色 念珠菌(C. albicans) 和对 3 种细菌: 大肠杆菌 (E. coli)、枯 草芽孢杆菌(B. subtilis)、金黄色葡萄球菌(S.aureus)的抑 菌活性, 结果如表 1 所示. 


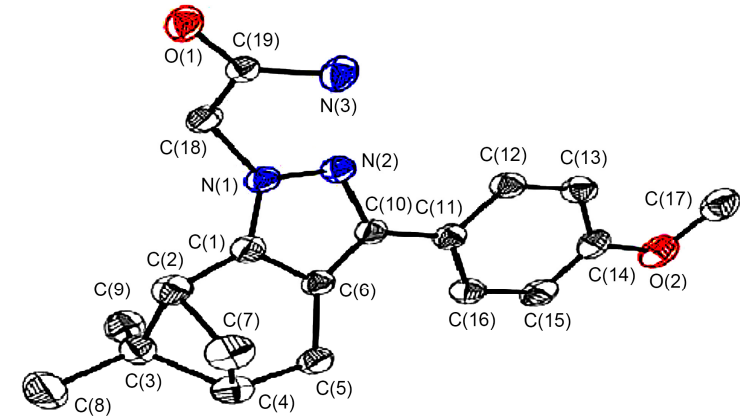

图 2 化合物 $\mathbf{4 d}$ 的分子结构图

Figure 2 Molecular structure of compound $\mathbf{4 d}$

由表 1 可知, 化合物 $\mathbf{3 a} \sim \mathbf{3 g}$ 无论是对真菌还是细菌 的抑菌活性都不太好, 但其酰胺化衍生物 $4 \mathrm{~b}, 4 \mathrm{c}$ 和 $4 \mathrm{~g}$ 的活性则有不同程度的增加. 化合物 $4 \mathrm{~b}$ 对热带假丝酵 母菌和金黄色葡萄球菌具有较好的抑制活性. 然而化合 物 $4 \mathrm{~g}$ 对真菌和细菌均表现出较好的抑制活性, 与阳性 对照的效果比较接近.

\section{4 杀虫活性}

采用浸渍法, 测定供试杀虫剂对紫薇蚜虫的毒力效 果, 噻虫嗪为阳性对照. 化合物浓度与试虫死亡率之间 的关系如表 2 所示. 对笁选出来的 3 个吡唑酰胺类化合 物, 处理蚜虫 $24 \mathrm{~h}$, 分别测定对紫薇蚜虫的杀虫活性. 采用概率单位回归法计算 $\mathrm{LC}_{50}$ ，结果如表 3 所示.

由表 2 可知，中间体蒎烷基吡唑类化合物 $3 \mathbf{a} \sim \mathbf{3 g}$ 对紫薇蚜虫表现出极弱的活性, 而目标化合物蒎烷基吡 唑酰胺类化合物 $4 \mathrm{a} \sim \mathbf{4 g}$ 对紫薇蚜虫有一定的活性，表 明酰胺基团的引入增强了该类化合物的活性. 由表 3 可 知，所笁选出的 3 种不同基团的吡唑酰胺类化合物，供 试浓度不同，杀虫活性程度也不一样，总体呈现浓度效 应，杀虫能力随浓度的增大而增大. 目标化合物 $\mathbf{4 a}$, 即 苯环上取代基为供电子基团甲基时，处理紫薇蚜虫 24 小时后，半数蚜虫死亡时的给药浓度 $16.73 \mathrm{mg}$ a.i./L; 目 标化合物 4d, 即苯环上取代基为对位甲氧基时, 处理紫 薇蚜虫 $24 \mathrm{~h}$ 后, 杀虫活性中等, 半数致死浓度在

表 1 化合物 3a $\sim 3 \mathrm{~g}$ 和 $4 \mathrm{a} \sim 4 \mathrm{~g}$ 的 MIC 值 $(\mu \mathrm{g} / \mathrm{mL})$

Table 1 MIC $(\mu \mathrm{g} / \mathrm{mL})$ of the compounds $3 \mathrm{a} \sim 3 \mathrm{~g}$ and $\mathbf{4 a} \sim \mathbf{4 g}$

\begin{tabular}{|c|c|c|c|c|c|c|c|}
\hline \multirow{2}{*}{ Compd. } & \multirow{2}{*}{$\mathrm{Ar}$} & \multicolumn{3}{|c|}{ Fungi } & \multicolumn{3}{|c|}{ Bacteria } \\
\hline & & G. tropicalis & A. niger & C. albicans & E. coli & B. subtilis & S. aureus \\
\hline $3 a$ & $4-\mathrm{CH}_{3}-\mathrm{C}_{6} \mathrm{H}_{4}$ & 31 & 16 & 63 & 16 & 16 & 63 \\
\hline $3 \mathbf{b}$ & 4-Cl- $\mathrm{C}_{6} \mathrm{H}_{4}$ & 31 & 4 & 63 & 31 & 16 & 16 \\
\hline $3 c$ & $4-\mathrm{Br}-\mathrm{C}_{6} \mathrm{H}_{4}$ & 31 & 31 & 16 & 31 & 8 & 16 \\
\hline 3d & 4- $\mathrm{CH}_{3} \mathrm{O}-\mathrm{C}_{6} \mathrm{H}_{4}$ & 31 & 63 & 63 & 16 & 31 & 16 \\
\hline $3 e$ & $2-\mathrm{Cl}-\mathrm{C}_{6} \mathrm{H}_{4}$ & 16 & 8 & 31 & 16 & 16 & 16 \\
\hline $3 f$ & $4-\mathrm{NO}_{2}-\mathrm{C}_{6} \mathrm{H}_{4}$ & 31 & 31 & 16 & 63 & 31 & 16 \\
\hline $3 g$ & $4-\mathrm{F}-\mathrm{C}_{6} \mathrm{H}_{4}$ & 31 & 31 & 31 & 31 & 16 & 16 \\
\hline $4 a$ & 4- $\mathrm{CH}_{3}-\mathrm{C}_{6} \mathrm{H}_{4}$ & 63 & 63 & 31 & 63 & 31 & 16 \\
\hline $4 \mathrm{~b}$ & $4-\mathrm{Cl}-\mathrm{C}_{6} \mathrm{H}_{4}$ & 4 & 63 & 31 & 16 & 31 & 8 \\
\hline $4 c$ & 4-Br- $\mathrm{C}_{6} \mathrm{H}_{4}$ & 8 & 4 & 31 & 8 & 16 & 63 \\
\hline 4d & $4-\mathrm{CH}_{3} \mathrm{O}-\mathrm{C}_{6} \mathrm{H}_{4}$ & 31 & 31 & 16 & 63 & 31 & 16 \\
\hline $4 e$ & $2-\mathrm{Cl}-\mathrm{C}_{6} \mathrm{H}_{4}$ & 31 & 16 & 63 & 16 & 31 & 31 \\
\hline $4 f$ & $4-\mathrm{NO}_{2}-\mathrm{C}_{6} \mathrm{H}_{4}$ & 63 & 31 & 31 & 63 & 31 & 4 \\
\hline $4 g$ & $4-\mathrm{F}-\mathrm{C}_{6} \mathrm{H}_{4}$ & 16 & 8 & 16 & 16 & 8 & 8 \\
\hline $\mathrm{PC}^{a}$ & & 4 & 4 & 2 & 4 & 1 & 2 \\
\hline
\end{tabular}

a阳性对照(positive control): 细菌为阿米卡星, 真菌为酮康唑.

表 2 化合物 $3 \mathrm{a} \sim 3 \mathrm{~g}$ 和 $4 \mathrm{a} \sim 4 \mathrm{~g}$ 对紫薇蚜虫的抑制活性

Table 2 Insecticidal activities of compounds $3 \mathbf{a} \sim 3 \mathbf{g}$ and $\mathbf{4 a} \sim \mathbf{4 g}$ against tinocallis kahawaluokalani

\begin{tabular}{ccc||ccc}
\hline Compd. & Concentration $/\left(\mathrm{mg} \cdot \mathrm{L}^{-1}\right)$ & Mortality $/ \%$ & Compd. & Concentration $/\left(\mathrm{mg} \cdot \mathrm{L}^{-1}\right)$ & Mortality $/ \%$ \\
\hline 3a & 200 & 4.4 & $\mathbf{4 a}$ & 200 & 97.2 \\
3b & 200 & 2.2 & $\mathbf{4 b}$ & 200 & 13.9 \\
$\mathbf{3 c}$ & 200 & 8.9 & $\mathbf{4 c}$ & 200 & 5.7 \\
$\mathbf{3 d}$ & 200 & 2.2 & $\mathbf{4 d}$ & 200 & 65.3 \\
$\mathbf{3 e}$ & 200 & 15.6 & $\mathbf{4 e}$ & 200 & 18.8 \\
$\mathbf{3 f}$ & 200 & 2.2 & $\mathbf{4 f}$ & 200 & 17.1 \\
$\mathbf{3 g}$ & 200 & 6.7 & $\mathbf{4 g}$ & 200 & 100.0 \\
噻虫嗪 & 200 & 100 & 噻虫嗪 & 200 & 100 \\
空白 & 0 & 2.2 & 空白 & 0 & 3.8 \\
\hline
\end{tabular}


表 3 化合物 $4 \mathrm{a}, 4 \mathrm{~d}$ 和 $4 \mathrm{~g}$ 对紫薇蚜虫的室内毒力 ${ }^{a}$

Table 3 Toxicity of compounds $4 \mathrm{a}, \mathbf{4 d}$, and $\mathbf{4 g}$ against tinocallis kahawaluokalani

\begin{tabular}{ccl}
\hline Compd. & Toxicity regression equation & \multicolumn{1}{c}{$\mathrm{LC}_{50}(95 \% \mathrm{~F} . \mathrm{L}) /.\left(\mathrm{mg}\right.$ a.i. $\left.\cdot \mathrm{L}^{-1}\right)$} \\
\hline $\mathbf{4 a}$ & $Y=2.3202+2.1901 X$ & $16.7337(11.7919 \sim 21.1155)$ \\
$\mathbf{4 d}$ & $Y=0.8082+1.9856 X$ & $129.1723(104.1566 \sim 157.8365)$ \\
$\mathbf{4 g}$ & $Y=3.8381+1.0312 X$ & $13.3896(4.3104 \sim 22.7585)$ \\
噻虫嗪 & $Y=4.7617+2.2154 X$ & $1.2811(1.0184 \sim 1.5396)$ \\
\hline
\end{tabular}

阳性对照(positive control)为噻虫溙, CK 为空白对照组.

104.15 157.83 mg a.i./L; 目标化合物 4g, 即苯环上取 代基为对氟时, 处理紫薇蚜虫 $24 \mathrm{~h}$ 后, 杀虫活性较好, 半数蚜虫死亡时的给药浓度 $13.38 \mathrm{mg}$ a.i./L. 本文合成 的 7 个新型蒎烷基吡唑酰胺衍生物中, 目标化合物中苯 环上取代基为甲基和氟时表现出较好的抗虫活性

\section{2 结论}

以诺蒎酮为先导化合物合成了新型蒎烷基吡唑酰 胺类衍生物. 采用 ${ }^{1} \mathrm{H} N M R,{ }^{13} \mathrm{C} N M R, I R, M S$ 和 HRMS 等分析手段表征所得化合物的结构, 并测试了化合物的 抑菌活性和抗虫活性. 生物活性测试结果表明, 吡唑酰 胺类化合物对不同菌种具有广谱抑菌活性, 是极具潜力 的抗真菌、抗细菌化合物; 化合物 $4 \mathrm{a}$ 和 $4 \mathrm{~g}$ 对紫薇蚜虫 有较好的杀虫效果.

\section{3 实验部分}

\section{1 仪器与试剂}

Nicolet 380 IR 红外光谱仪 ( $\mathrm{KBr}$ 压片法); 德国 Bruker AV 500/600 MHz 核磁共振仪 (TMS 为内标, DMSO 为溶剂); 美国 Agilent 7890A/5975 气相质谱联用 仪, 北京泰克 X-6 显微熔点测定仪 (温度计未经校正); 德国 Bruker 高分辨质谱仪. $\beta$-蒎烯购自江西省吉水县宏 达天然香料有限公司. 其余试剂均为分析纯或化学纯.

\section{2 实验方法}

\subsection{1 中间体 3-芳亚甲基诺蒎酮 $\mathbf{2} \mathbf{a} \sim 2 \mathrm{~g}$ 的合成}

将 $10 \mathrm{mmol}$ 诺蒎酮 ${ }^{[22]}$ 、30 $\mathrm{mL}$ 叔丁醇、 $12 \mathrm{mmol}$ 对 甲基苯甲醛和 $56 \mathrm{mmol}$ 甲醇钠依次加入配有摚拌器、温 度计和回流冷凝器的 $100 \mathrm{~mL}$ 三口烧瓶中, 电磁搅拌下 加热至回流反应数小时, 至诺蒎酮转化率达 $95 \%$ 以上 (GC 跟踪检测). 反应液用乙酸乙酯萃取, 经饱和食盐水 洗至中性, 无水 $\mathrm{Na}_{2} \mathrm{SO} 4$ 干燥、过滤、浓缩后得到粗产 物, 再经乙醇重结晶得到产物 $2 \mathbf{a}$. 采用同样方法得到 $\mathbf{2 b} \sim \mathbf{2 g}$.

3-(4'-甲基亚芳基)诺蒎酮(2a) ${ }^{[23]}$ : 得率 $85.7 \%$, 无色 透明晶体. m.p. 95 96 ${ }^{\circ} \mathrm{C} ;{ }^{1} \mathrm{H}$ NMR $\left(\mathrm{CDCl}_{3}, 600 \mathrm{MHz}\right)$ $\delta$ : $0.93(\mathrm{~s}, 3 \mathrm{H}), 1.38(\mathrm{~s}, 3 \mathrm{H}), 1.50(\mathrm{~d}, J=10.2 \mathrm{~Hz}, 1 \mathrm{H})$, $2.35 \sim 2.37(\mathrm{~m}, 1 \mathrm{H}), 2.38(\mathrm{~s}, 3 \mathrm{H}), 2.60 \sim 2.64(\mathrm{~m}, 1 \mathrm{H})$,
2.69 (t, $J=5.4 \mathrm{~Hz}, 1 \mathrm{H}), 2.96(\mathrm{~d}, J=2.4 \mathrm{~Hz}, 2 \mathrm{H}), 7.22$ (d, $J=8.4 \mathrm{~Hz}, 2 \mathrm{H}), 7.49(\mathrm{~d}, J=7.8 \mathrm{~Hz}, 2 \mathrm{H}), 7.68(\mathrm{~s}, 1 \mathrm{H}) ;{ }^{13} \mathrm{C}$ NMR $\left(\mathrm{CDCl}_{3}, 150 \mathrm{MHz}\right) \delta: 21.73,21.99,26.59,27.88$, 31.36, 39.85, 41.21, 56.27, 129.69, 131.20, 132.10, 133.34, 136.09, 139.57, 203.89; IR (KBr) v: 2951, 2927, 2881, $1682,1596,1507,1439,1047,928,840,813 \mathrm{~cm}^{-1}$; MS (70 eV) $m / z(\%): 240\left(\mathrm{M}^{+}, 100\right), 225$ (93), 197 (55).

3-(4'-氯亚茮基)诺蒎酮(2b): 得率 $85.5 \%$, 无色透明 晶体. m.p. $109 \sim 110{ }^{\circ} \mathrm{C} ;{ }^{1} \mathrm{H}$ NMR $\left(\mathrm{CDCl}_{3}, 600 \mathrm{MHz}\right) \delta$ : $0.93(\mathrm{~s}, 3 \mathrm{H}), 1.39$ (s, 3H), $1.50(\mathrm{~d}, J=10.8 \mathrm{~Hz}, 1 \mathrm{H}), 2.36 \sim$ $2.38(\mathrm{~m}, 1 \mathrm{H}), 2.62 \sim 2.66(\mathrm{~m}, 1 \mathrm{H}), 2.71(\mathrm{t}, J=6.0 \mathrm{~Hz}, 1 \mathrm{H})$, 2.94 (s, 2H), 7.38 (d, $J=8.4 \mathrm{~Hz}, 2 \mathrm{H}), 7.51(\mathrm{~d}, J=8.4 \mathrm{~Hz}$, $2 \mathrm{H}), 7.64(\mathrm{~s}, 1 \mathrm{H}) ;{ }^{13} \mathrm{C} \mathrm{NMR}\left(\mathrm{CDCl}_{3}, 150 \mathrm{MHz}\right) \delta: 20.65$, 25.21, 26.45, 29.88, 38.38, 39.93, 54.87, 127.83, 130.88, $132.18,133.19,133.32,133.86,202.12$; IR (KBr) v: 2951, $2872,1686,1600,1490,1405,1255,1216,1051,824$ $\mathrm{cm}^{-1}$; MS (70 eV) m/z (\%): $260\left(\mathrm{M}^{+}, 100\right), 245$ (49), 217 (49).

3-(4'-溴亚茮基)诺蒎酩(2c)：得率 $83.9 \%$, 无色透明 晶体. m.p. $140 \sim 141{ }^{\circ} \mathrm{C} ;{ }^{1} \mathrm{H}$ NMR $\left(\mathrm{CDCl}_{3}, 600 \mathrm{MHz}\right) \delta$ : $0.93(\mathrm{~s}, 3 \mathrm{H}), 1.39$ (s, 3H), 1.50 (d, $J=10.2 \mathrm{~Hz}, 1 \mathrm{H}), 2.35 \sim$ $2.38(\mathrm{~m}, 1 \mathrm{H}), 2.62 \sim 2.66(\mathrm{~m}, 1 \mathrm{H}), 2.71(\mathrm{t}, J=6.0 \mathrm{~Hz}, 1 \mathrm{H})$, 2.93 (s, 2H), 7.44 (d, $J=8.4 \mathrm{~Hz}, 2 \mathrm{H}), 7.54$ (d, $J=8.4 \mathrm{~Hz}$, 2H), $7.62(\mathrm{~s}, 1 \mathrm{H}) ;{ }^{13} \mathrm{C} \mathrm{NMR}\left(\mathrm{CDCl}_{3}, 150 \mathrm{MHz}\right) \delta: 21.72$, 26.28, 27.52, 30.96, 39.44, 41.02, 55.95, 123.28, 131.87, $132.17,133.43,134.45,134.68,203.20$; IR (KBr) v: 2950, 2871, 1684, 1600, 1484, 1401, 1296, 1188, 1002, 823 $\mathrm{cm}^{-1}$; MS (70 eV) m/z (\%): $304\left(\mathrm{M}^{+}, 84\right), 289$ (42), 263 (48).

3-(4'-甲氧基亚苄基)诺蒎酮(2d): 得率 83.5\%, 无色 透明晶体. m.p. $82 \sim 83{ }^{\circ} \mathrm{C} ;{ }^{1} \mathrm{H}$ NMR $\left(\mathrm{CDCl}_{3}, 600 \mathrm{MHz}\right)$ $\delta: 0.93(\mathrm{~s}, 3 \mathrm{H}), 1.38(\mathrm{~s}, 3 \mathrm{H}), 1.51(\mathrm{~d}, J=10.2 \mathrm{~Hz}, 1 \mathrm{H})$, $2.36 \sim 2.38(\mathrm{~m}, 1 \mathrm{H}), 2.60 \sim 2.63(\mathrm{~m}, 1 \mathrm{H}), 2.69(\mathrm{t}, J=6.0$ $\mathrm{Hz}, 1 \mathrm{H}), 2.96$ (t, $J=3.0 \mathrm{~Hz}, 2 \mathrm{H}), 3.85$ (s, 3H), 6.94 (d, $J=$ $8.4 \mathrm{~Hz}, 2 \mathrm{H}), 7.57$ (d, $J=8.4 \mathrm{~Hz}, 2 \mathrm{H}), 7.67(\mathrm{~s}, 1 \mathrm{H}) ;{ }^{13} \mathrm{C}$ NMR $\left(\mathrm{CDCl}_{3}, 150 \mathrm{MHz}\right) \delta: 21.64,26.26,27.62,31.05$, $39.55,40.84,55.35,55.91,114.13,128.58,130.35,132.62$, 135.45, 160.23, 203.60; IR (KBr) v: 2964, 2931, 2838, 
$1682,1594,1511,1460,1421,1253,1028,829 \mathrm{~cm}^{-1}$; MS (70 eV) $m / z(\%): 256\left(\mathrm{M}^{+}, 100\right), 255$ (14), 241 (19).

3-(2'-氯亚苄基)诺蒎酮(2e): 得率 $90.9 \%$, 无色透明 晶体. m.p. $107 \sim 108{ }^{\circ} \mathrm{CC} ;{ }^{1} \mathrm{H} \mathrm{NMR}\left(\mathrm{CDCl}_{3}, 600 \mathrm{MHz}\right) \delta$ : $0.95(\mathrm{~s}, 3 \mathrm{H}), 1.38(\mathrm{~s}, 3 \mathrm{H}), 1.51(\mathrm{~d}, J=10.8 \mathrm{~Hz}, 1 \mathrm{H}), 2.29 \sim$ $2.31(\mathrm{~m}, 1 \mathrm{H}), 2.60 \sim 2.64(\mathrm{~m}, 1 \mathrm{H}), 2.72(\mathrm{t}, J=5.4 \mathrm{~Hz}, 1 \mathrm{H})$, $2.79 \sim 2.86(\mathrm{~m}, 2 \mathrm{H}), 7.26 \sim 7.29(\mathrm{~m}, 2 \mathrm{H}), 7.43 \sim 7.45(\mathrm{~m}$, $1 \mathrm{H}), 7.50 \sim 7.52(\mathrm{~m}, 1 \mathrm{H}), 7.91(\mathrm{~s}, 1 \mathrm{H}) ;{ }^{13} \mathrm{C} \mathrm{NMR}\left(\mathrm{CDCl}_{3}\right.$, $150 \mathrm{MHz}) \delta: 21.71,26.25,27.21,29.82,39.28,41.13$, $55.95,126.40,129.57,129.91,129.97,132.61,134.17$, 135.00, 135.21, 203.01; IR (KBr) v: 2989, 2954, 1693, $1609,1467,1433,1054,1038,772,749 \mathrm{~cm}^{-1}$; MS (70 eV) m/z (\%): 260 ( $\left.\mathrm{M}^{+}, 13\right), 245$ (14), 225 (100).

3-(4'-硝基亚苠基)诺蒎酮(2f): 得率 $62.7 \%$, 黄色透 明晶体. m.p. $151 \sim 152{ }^{\circ} \mathrm{C} ;{ }^{1} \mathrm{H}$ NMR $\left(\mathrm{CDCl}_{3}, 600 \mathrm{MHz}\right)$ $\delta: 0.94(\mathrm{~s}, 3 \mathrm{H}), 1.41(\mathrm{~s}, 3 \mathrm{H}), 1.52(\mathrm{~d}, J=10.8 \mathrm{~Hz}, 1 \mathrm{H})$, $2.39 \sim 2.41(\mathrm{~m}, 1 \mathrm{H}), 2.66 \sim 2.70(\mathrm{~m}, 1 \mathrm{H}), 2.76(\mathrm{t}, J=5.4$ $\mathrm{Hz}, 1 \mathrm{H}), 2.99$ (s, 2H), 7.72 (d, $J=9.0 \mathrm{~Hz}, 3 \mathrm{H}), 8.26$ (d, $J=$ $8.4 \mathrm{~Hz}, 2 \mathrm{H}) ;{ }^{13} \mathrm{C} \mathrm{NMR}\left(\mathrm{CDCl}_{3}, 150 \mathrm{MHz}\right) \delta: 21.80,26.28$, 27.47, 30.99, 39.34, 41.18, 55.98, 123.83, 131.13, 133.04, 136.53, 142.15, 147.40, 202.61; IR (KBr) v: 2974, 2966, 2922, 1689, 1610, 1516, 1464, 1343, 1044, 982, $853 \mathrm{~cm}^{-1}$; MS (70 eV) m/z (\%): $271\left(\mathrm{M}^{+}, 100\right), 254$ (490), 228 (37).

3-(4'-氟亚茮基)诺蒎酮(2g): 得率 $90.0 \%$, 无色透明 晶体, m.p. 91 92 ${ }^{\circ} \mathrm{C}$; ${ }^{1} \mathrm{H}$ NMR $\left(\mathrm{CDCl}_{3}, 600 \mathrm{MHz}\right) \delta$ : $0.93(\mathrm{~s}, 3 \mathrm{H}), 1.39(\mathrm{~s}, 3 \mathrm{H}), 1.51(\mathrm{~d}, J=10.8 \mathrm{~Hz}, 1 \mathrm{H}), 2.35 \sim$ $2.41(\mathrm{~m}, 1 \mathrm{H}), 2.61 \sim 2.65(\mathrm{~m}, 1 \mathrm{H}), 2.71(\mathrm{t}, J=6.0 \mathrm{~Hz}, 1 \mathrm{H})$, $2.94(\mathrm{~s}, 2 \mathrm{H}), 7.09 \sim 7.12(\mathrm{~m}, 2 \mathrm{H}), 7.56 \sim 7.59(\mathrm{~m}, 2 \mathrm{H})$, $7.67(\mathrm{~s}, 1 \mathrm{H}) ;{ }^{13} \mathrm{C} \mathrm{NMR}\left(\mathrm{CDCl}_{3}, 150 \mathrm{MHz}\right) \delta: 21.96,26.54$, 27.80, 31.16, 39.74, 41.24, 56.20, 115.52, 115.94, 116.08, $132.32,132.56,132.92,132.98,134.80,162.32,163.99$, 203.58; IR (KBr) v: 2958, 2933, 2868, 1690, 1615, 1507, 1439, 1096, 1055, $838 \mathrm{~cm}^{-1}$; MS (70 eV) m/z (\%): 244 $\left(\mathrm{M}^{+}, 100\right), 229$ (50), 201 (58).

\subsection{2中间体 $\mathbf{3 a} \sim \mathbf{3 g}$ 的合成通法}

在单口烧瓶中，依次加入 $15 \mathrm{mmol}$ 中间体 $\mathbf{2 a} \sim \mathbf{2 g}$ 、 $75 \mathrm{mmol}$ 水合肼 $(80 \%) 、 90 \mathrm{~mL}$ 无水乙醇, 电磁搅拌下加 热升温回流反应 $10 \mathrm{~h}$. 反应液冷却至室温后, 分两次加 入适量乙醇, 油浴旋蒸除去反应液中的溶剂和过量的水 合肼; 旋蒸后, 再依次加入 $90 \mathrm{~mL}$ 干燥的 1,4-二氧六环、 $16.5 \mathrm{mmol} 2,3$-二氯-5,6-二氰对苯醌(DDQ), 搅拌放热 后, 回流反应 6 $8 \mathrm{~h}$; 然后冷却至室温, 加入 $45 \mathrm{~mL}$ 质 量分数为 $20 \% \mathrm{KOH}$ 水溶液、适量的亚硫酸钠水溶液、 $90 \mathrm{~mL}$ 二氯甲烷, 室温摚拌 $0.5 \mathrm{~h}$, 再用二氯甲烷萃取, 合并有机相用去离子水洗至中性; 经无水硫酸钠干燥、
过滤、浓缩后得到油状初产物, 采用硅胶色谱柱纯化 $[V($ 石油醚 $): V($ 乙酸乙酯 $)=3: 1]$, 甲醇或乙腈重结晶, 得到目标化合物 $\mathbf{3 a} \sim \mathbf{3 g}$.

6,6-二甲基-3-对甲苯基-4,5,6,7-四氢- $2 H$-5,7-桥亚甲 基吲唑(3a): 得率为 $73.5 \%$, 浅灰色粉末状固体. m.p. $166 \sim 168{ }^{\circ} \mathrm{C}$; ${ }^{1} \mathrm{H}$ NMR (DMSO- $\left.d_{6}, 600 \mathrm{MHz}\right) \delta$ : 0.65 (s, $3 \mathrm{H}), 1.23 \sim 1.28(\mathrm{~m}, 1 \mathrm{H}), 1.39(\mathrm{~s}, 3 \mathrm{H}), 2.29(\mathrm{~s}, 1 \mathrm{H}), 2.32$ $(\mathrm{s}, 3 \mathrm{H}), 2.68 \sim 2.71(\mathrm{~m}, 1 \mathrm{H}), 2.80(\mathrm{t}, J=15 \mathrm{~Hz}, 1 \mathrm{H}), 2.82$ (d, $J=13.8 \mathrm{~Hz}, 2 \mathrm{H}), 7.25$ (d, $J=6.6 \mathrm{~Hz}, 2 \mathrm{H}), 7.57$ (d, $J=$ $6.0 \mathrm{~Hz}, 2 \mathrm{H}), 12.31$ (brs, $1 \mathrm{H}) ;{ }^{13} \mathrm{C}$ NMR (DMSO- $d_{6}, 150$ MHz) $\delta: 21.24,25.85,26.03,32.05,40.42$, 41.16, 107.87, $124.85,127.87,129.26,136.27,158.77$; IR (KBr) v: 3116 , 3056, 2965, 2939, 2907, 1517, 1446, 1121, 983, 816, 662 $\mathrm{cm}^{-1}$; MS (70 eV) m/z (\%): $252\left(\mathrm{M}^{+}, 33\right), 237$ (37), 209 (100); ESI-HRMS calcd for $\mathrm{C}_{17} \mathrm{H}_{21} \mathrm{~N}_{2}[\mathrm{M}+\mathrm{H}]^{+}$253.1699, found 253.1709 .

6,6-二甲基-3-对氯苯基-4,5,6,7-四氢-2H-5,7-桥亚甲 基吲唑(3b): 得率为 83.2\%, 白色棉状固体. m.p. 198 $199{ }^{\circ} \mathrm{C} ;{ }^{1} \mathrm{H}$ NMR (DMSO- $\left.d_{6}, 600 \mathrm{MHz}\right) \delta: 0.64$ (s, 3H), $1.23 \sim 1.28(\mathrm{~m}, 1 \mathrm{H}), 1.39(\mathrm{~s}, 3 \mathrm{H}), 2.30(\mathrm{~s}, 1 \mathrm{H}), 2.69 \sim 2.72$ (m, 1H), 2.78 (s, 1H), 2.82 (d, $J=15.6 \mathrm{~Hz}, 1 \mathrm{H}), 2.93$ (d, $J=15.6 \mathrm{~Hz}, 1 \mathrm{H}), 7.51(\mathrm{~d}, J=6.6 \mathrm{~Hz}, 2 \mathrm{H}), 7.69$ (d, $J=6.6$ $\mathrm{Hz}, 2 \mathrm{H}), 12.46$ (brs, $1 \mathrm{H}) ;{ }^{13} \mathrm{C}$ NMR (DMSO- $d_{6}, 150 \mathrm{MHz}$ ) $\delta: 21.21,25.77,25.95,31.97,38.93,40.93,108.61,126.59$, 128.72, 130.35, 131.48, 137.65, 156.91; IR (KBr) v: 3127, 3048, 2970, 2940, 1493, 1444, 1120, 1090, 982, $827 \mathrm{~cm}^{-1}$; MS (70 eV) m/z (\%): $272\left(\mathrm{M}^{+}, 24\right), 257$ (32), 229 (100); ESI-HRMS calcd for $\mathrm{C}_{16} \mathrm{H}_{18} \mathrm{ClN}_{2}[\mathrm{M}+\mathrm{H}]^{+}$273.1153, found 273.1171 .

6,6-二甲基-3-对溴苯基-4,5,6,7-四氢-2H-5,7-桥亚甲 基唑 (3c): 得率为 $80.2 \%$, 褐色颗粒状固体. m.p. $203 \sim 204{ }^{\circ} \mathrm{C}$; ${ }^{1} \mathrm{H}$ NMR (DMSO- $\left.d_{6}, 600 \mathrm{MHz}\right) \delta$ : 0.68 (s, $3 \mathrm{H}), 1.26 \sim 1.28(\mathrm{~m}, 1 \mathrm{H}), 1.39(\mathrm{~s}, 3 \mathrm{H}), 2.23 \sim 2.30(\mathrm{~m}$, $1 \mathrm{H}), 2.68 \sim 2.72(\mathrm{~m}, 1 \mathrm{H}), 2.79(\mathrm{~s}, 1 \mathrm{H}), 2.82(\mathrm{~d}, J=15.6$ $\mathrm{Hz}, 1 \mathrm{H}), 2.93$ (d, $J=15.6 \mathrm{~Hz}, 1 \mathrm{H}), 7.63$ (s, 4H), 12.46 (brs, $1 \mathrm{H}) ;{ }^{13} \mathrm{C}$ NMR (DMSO- $\left.d_{6}, 150 \mathrm{MHz}\right) \delta: 21.23,25.78$, 25.96, 31.98, 38.93, 40.91, 108.67, 119.98, 126.90, 130.68, 131.64, 137.55, 156.88; IR (KBr) v: 3125, 3044, 2911, $1488,1444,1296,1120,1070,1010,979,822 \mathrm{~cm}^{-1}$; MS (70 eV) $m / z(\%): 318\left(\mathrm{M}^{+}, 100\right), 301$ (32), 287 (8), 273 (100); ESI-HRMS calcd for $\mathrm{C}_{16} \mathrm{H}_{18} \mathrm{BrN}_{2}[\mathrm{M}+\mathrm{H}]^{+}$ 317.0647 , found 317.0658 .

6,6-二甲基-3-(对-甲氧基苯基)-4,5,6,7-四氢- $2 H-5,7$ 桥亚甲基吲唑(3d): 得率为 78.3\%, 黄色砂状固体. m.p. $145 \sim 146{ }^{\circ} \mathrm{C} ;{ }^{1} \mathrm{H}$ NMR (DMSO- $\left.d_{6}, 600 \mathrm{MHz}\right) \delta$ : 0.65 (s, 
$3 \mathrm{H}), 1.26 \sim 1.28(\mathrm{~m}, 1 \mathrm{H}), 1.38(\mathrm{~s}, 3 \mathrm{H}), 2.29((\mathrm{~d}, J=1.8 \mathrm{~Hz}$, $1 \mathrm{H}), 2.69$ (t, $J=5.4 \mathrm{~Hz}, 1 \mathrm{H}), 2.75 \sim 2.81(\mathrm{~m}, 2 \mathrm{H}), 2.90(\mathrm{~d}$, $J=15.0 \mathrm{~Hz}, 1 \mathrm{H}), 3.78$ (d, $J=1.8 \mathrm{~Hz}, 3 \mathrm{H}), 7.01$ (d, $J=8.4$ $\mathrm{Hz}, 2 \mathrm{H}), 7.61$ (d, $J=8.4 \mathrm{~Hz}, 2 \mathrm{H}), 12.22$ (brs, $1 \mathrm{H}) ;{ }^{13} \mathrm{C}$ NMR (DMSO- $\left.d_{6}, 150 \mathrm{MHz}\right) \delta: 21.25,25.76,26.03,32.04$, 38.92 , 41.12, 55.04, 107.26, 113.72, 114.18, 123.87, 126.28, 127.02, 137.79, 157.48, 158.34; IR (KBr) v: 3148, 3061, 2923, 1603, 1517, 1448, 1233, 981, 837, $814 \mathrm{~cm}^{-1}$; MS (70 eV) m/z (\%): $268\left(\mathrm{M}^{+}, 56\right), 253$ (44), 225 (100); ESI-HRMS calcd for $\mathrm{C}_{17} \mathrm{H}_{21} \mathrm{~N}_{2} \mathrm{O}[\mathrm{M}+\mathrm{H}]^{+}$269.1648, found 269.1657 .

6,6-二甲基-3-邻氯苯基-4,5,6,7-四氢- $2 H$-5,7-桥亚甲 基吲唑 (3e): 得率为 $81.1 \%$, 棕色颗粒状固体. m.p. $159 \sim 160{ }^{\circ} \mathrm{C}$; ${ }^{1} \mathrm{H}$ NMR (DMSO- $\left.d_{6}, 600 \mathrm{MHz}\right) \delta$ : 0.68 (s, $3 \mathrm{H}), 1.38(\mathrm{~s}, 3 \mathrm{H}), 1.26 \sim 1.28(\mathrm{~m}, 1 \mathrm{H}), 2.24(\mathrm{~s}, 1 \mathrm{H}), 2.70$ (t, $J=9.6 \mathrm{~Hz}, 1 \mathrm{H}), 2.82(\mathrm{~d}, J=15.6 \mathrm{~Hz}, 2 \mathrm{H}), 3.30(\mathrm{~s}, 1 \mathrm{H})$, 7.37 (s, 1H), 7.43 (d, $J=3.0 \mathrm{~Hz}, 1 \mathrm{H}), 7.47$ (t, $J=4.8 \mathrm{~Hz}$, 1H), 7.57 (t, $J=4.2 \mathrm{~Hz}, 1 \mathrm{H}), 12.15$ (brs, $1 \mathrm{H}) ;{ }^{13} \mathrm{C} \mathrm{NMR}$ $\left(\mathrm{DMSO}-d_{6}, 150 \mathrm{MHz}\right) \delta: 21.32,24.76,26.05,32.26,38.94$, $40.88,41.00,110.45,127.01,129.65,129.70,130.65$, 131.42, 131.89; IR (KBr) v: 3115, 3038, 2933, 1604, 1489, 1439, 1255, 1123, 979, 861, 756, $672 \mathrm{~cm}^{-1}$; MS (70 eV) m/z (\%): $272\left(\mathrm{M}^{+}, 15\right), 257$ (30), 229 (100); ESI-HRMS calcd for $\mathrm{C}_{16} \mathrm{H}_{18} \mathrm{ClN}_{2}[\mathrm{M}+\mathrm{H}]^{+}$273.1153, found 273.1166 .

6,6-二甲基-3-对硝基苯基-4,5,6,7-四氢- $2 H-5,7$-桥亚 甲基吲唑 $(\mathbf{3 f})$ : 得率为 $82.4 \%$, 淡黄色棉状固体. m.p. $198 \sim 201{ }^{\circ} \mathrm{C} ;{ }^{1} \mathrm{H}$ NMR (DMSO- $\left.d_{6}, 600 \mathrm{MHz}\right) \delta$ : 0.65 (s, $3 \mathrm{H}), 1.40(\mathrm{~s}, 3 \mathrm{H}), 1.23(\mathrm{~s}, 1 \mathrm{H}), 2.33(\mathrm{~s}, 1 \mathrm{H}), 2.70(\mathrm{t}, J=$ $15.0 \mathrm{~Hz}, 1 \mathrm{H}), 2.78$ (t, $J=18.0 \mathrm{~Hz}, 1 \mathrm{H}), 2.82(\mathrm{~d}, J=15.0$ $\mathrm{Hz}, 2 \mathrm{H}), 7.93(\mathrm{~d}, J=7.2 \mathrm{~Hz}, 2 \mathrm{H}), 8.27 \sim 8.32(\mathrm{~m}, 2 \mathrm{H})$, 12.78 (brs, $1 \mathrm{H}) ;{ }^{13} \mathrm{C}$ NMR (DMSO- $\left.d_{6}, 150 \mathrm{MHz}\right) \delta: 21.19$, 25.86, 25.97, 31.19, 38.94, 40.93, 110.47, 124.11, 125.62, 138.24, 145.68, 155.73; IR (KBr) v: 3128, 3039, 2944, 2902, 1603, 1516, 1339, 1111, 979, 852, $691 \mathrm{~cm}^{-1}$; MS (70 eV) $m / z(\%): 283\left(\mathrm{M}^{+}, 20\right), 268$ (24), 240 (100); ESI-HRMS calcd for $\mathrm{C}_{16} \mathrm{H}_{18} \mathrm{~N}_{3} \mathrm{O}_{2}[\mathrm{M}+\mathrm{H}]^{+}$284.1393, found 284.1403 .

6,6-二甲基-3-对氟苯基-4,5,6,7-四氢- $2 H-5,7$-桥亚甲 基吲唑 $(3 \mathrm{~g})$ : 得率为 $79.5 \%$, 白色颗粒状固体. m.p. $168 \sim 169{ }^{\circ} \mathrm{C} ;{ }^{1} \mathrm{H}$ NMR (DMSO- $\left.d_{6}, 600 \mathrm{MHz}\right) \delta$ : 0.65 (s, $3 \mathrm{H}), 1.39(\mathrm{~s}, 3 \mathrm{H}), 1.23 \sim 1.27(\mathrm{~m}, 1 \mathrm{H}), 2.30(\mathrm{~s}, 1 \mathrm{H}), 2.91 \sim$ $2.94(\mathrm{~m}, 1 \mathrm{H}), 3.17(\mathrm{~d}, J=5.4 \mathrm{~Hz}, 2 \mathrm{H}), 4.08$ (d, $J=4.8 \mathrm{~Hz}$, $1 \mathrm{H}$ ), 7.29 (d, $J=7.2 \mathrm{~Hz}, 2 \mathrm{H}), 7.71$ (s, 2H), 12.38 (brs, 1H); ${ }^{13} \mathrm{C}$ NMR (DMSO- $\left.d_{6}, 150 \mathrm{MHz}\right) \delta: 21.21,25.69,25.96$, 31.98 , 38.92, 40.93, 108.07, 115.58, 126.97, 135.89,
157.87, 159.50, 162.74; IR (KBr) v: 3148, 3061, 2923, $1603,1517,1448,1233,1158,1116,981,837,814 \mathrm{~cm}^{-1}$; MS (70 eV) m/z (\%): $256\left(\mathrm{M}^{+}, 25\right), 241$ (33), 227 (8), 213 (100); ESI-HRMS calcd for $\mathrm{C}_{16} \mathrm{H}_{18} \mathrm{FN}_{2}[\mathrm{M}+\mathrm{H}]{ }^{+}$ 257.1448 , found 257.1460 .

\subsection{3 目标化合物 $\mathbf{4 a} \sim \mathbf{4 g}$ 的合成通法}

将 $0.01 \mathrm{~mol}$ 吡唑 $\mathbf{3 a} \sim \mathbf{3 g}$ 溶于 $25 \mathrm{ml} \mathrm{DMF}$ 中, 加入 配有温度计、搅拌器和冷凝器的 $100 \mathrm{~mL}$ 三口烧瓶中, 加 入 $0.3 \mathrm{~g} \mathrm{NaOH}$ 固体, 加热至 $110{ }^{\circ} \mathrm{C}$ 并磁力摚拌 $30 \mathrm{~min}$ 后, 通过恒压漏斗向反应液中慢慢滴加 2-氯乙酰胺 $0.014 \mathrm{~mol}$ 的 $10 \mathrm{~mL} \mathrm{DMF}$ 溶液, 保持温度 $110{ }^{\circ} \mathrm{C}$ 不变回 流反应 $30 \mathrm{~h}$ (TLC 跟踪). 冷却至室温过滤后, 将滤液旋 干并加入乙酸乙酯饱和食盐水洗至中性, 有机层经无水 硫酸钠干燥后, 过滤并旋蒸, 所得粗产物使用 $V($ 石油 醚) $: V($ 乙酸乙酯 $)=1: 1$ 的展开剂柱色谱分离, 用 $V$ (石 油醚) $: V($ 乙酸乙酯 $)=3: 1$ 重结晶, 得到目标化合物 $\mathbf{4 a} \sim \mathbf{4 g}$.

2-[6 $6^{\prime}, 6^{\prime}$-二甲基- $3^{\prime}$-(4' - 甲基苯基)-4', $5^{\prime}, 6^{\prime}, 7^{\prime}$ - 四氢$5^{\prime}, 7^{\prime}$-桥亚甲基-吲唑-1'-基]乙酰胺(4a)：得率为 73.8\%, 棕黄色粉末状固体. m.p.129 $130{ }^{\circ} \mathrm{C} ;{ }^{1} \mathrm{H} \mathrm{NMR}$ (DMSO- $\left.d_{6}, 500 \mathrm{MHz}\right) \delta$ : $0.72(\mathrm{~s}, 3 \mathrm{H}), 1.41(\mathrm{~s}, 3 \mathrm{H}), 1.34 \sim$ $1.36(\mathrm{~m}, 1 \mathrm{H}), 2.33(\mathrm{~d}, J=4.0 \mathrm{~Hz}, 3 \mathrm{H}), 2.33(\mathrm{~s}, 1 \mathrm{H}), 2.53$ $(\mathrm{s}, 1 \mathrm{H}), 2.70 \sim 2.72(\mathrm{~m}, 1 \mathrm{H}), 2.82 \sim 2.97(\mathrm{~m}, 2 \mathrm{H}), 4.68(\mathrm{~s}$, 2H), $7.23(\mathrm{~d}, J=4.0 \mathrm{~Hz}, 2 \mathrm{H}), 7.67$ (d, $J=4.0 \mathrm{~Hz}, 2 \mathrm{H}), 7.21$ $(\mathrm{s}, 1 \mathrm{H}), 7.38(\mathrm{~s}, 1 \mathrm{H}) ;{ }^{13} \mathrm{C}$ NMR (DMSO-d, $\left.125 \mathrm{MHz}\right) \delta$ : 20.77, 21.24, 25.93, 26.12, 31.91, 40.63, 41.17, 50.94, $108.29,125.26,129.02,131.68,135.87,145.35,150.57$, 168.74; IR (KBr) v: 3466, 3281, 3156, 2913, 1676, 1611, 1539, 1383,943, 823, $728 \mathrm{~cm}^{-1}$; MS (70 eV) m/z (\%): 309 $\left(\mathrm{M}^{+}, 68\right), 294$ (15), 280 (7), 266 (48); ESI-HRMS calcd for $\mathrm{C}_{19} \mathrm{H}_{24} \mathrm{~N}_{3} \mathrm{O}[\mathrm{M}+\mathrm{H}]^{+}$310.1913, found 310.1924.

2-[6', $6^{\prime}$-二甲基- $3^{\prime}$-( $4^{\prime}$-氯苯基)-4', $5^{\prime}, 6^{\prime}, 7^{\prime}$-四氢-5', $7^{\prime}$ 桥亚甲基-吲唑- $1^{\prime}$-基]乙酰胺 (4b): 得率为 $72.5 \%$, 棕黄 色粉末状固体. m.p.144 $145{ }^{\circ} \mathrm{C} ;{ }^{1} \mathrm{H}$ NMR (DMSO- $d_{6}$, $500 \mathrm{MHz}) \delta: 0.71(\mathrm{~s}, 3 \mathrm{H}), 1.41(\mathrm{~s}, 3 \mathrm{H}), 1.34 \sim 1.36(\mathrm{~m}$, $1 \mathrm{H}), 2.33(\mathrm{~s}, 1 \mathrm{H}), 2.53(\mathrm{~s}, 1 \mathrm{H}), 2.70 \sim 2.72(\mathrm{~m}, 1 \mathrm{H})$, 2.82 2.97 (m, 2H), $4.70(\mathrm{~s}, 2 \mathrm{H}), 7.47(\mathrm{~d}, J=4.3 \mathrm{~Hz}, 2 \mathrm{H})$, $7.78(\mathrm{~d}, J=4.3 \mathrm{~Hz}, 2 \mathrm{H}), 7.20(\mathrm{~s}, 1 \mathrm{H}), 7.42(\mathrm{~s}, 1 \mathrm{H}) ;{ }^{13} \mathrm{C}$ NMR (DMSO- $\left.d_{6}, 125 \mathrm{MHz}\right) \delta: 21.23,25.89,25.97,31.84$, $40.61,41.07,51.00,108.76,126.94,128.50,131.20$, 133.25, 144.14, 150.95, 168.54; IR (KBr) v: 3329, 3180, 2930, 2839, 1687, 1633, 1510, 1401, 1364, 1322, 832 $\mathrm{cm}^{-1}$; MS (70 eV) m/z (\%): $329\left(\mathrm{M}^{+}, 52\right), 314$ (16), 300 (6), 286 (46); ESI-HRMS calcd for $\mathrm{C}_{18} \mathrm{H}_{20} \mathrm{ClN}_{3} \mathrm{NaO}[\mathrm{M}+$ $\mathrm{Na}]^{+}$352.1187, found 352.1200. 
2-[6', 6'-二甲基-3'-(4' - 溴苯基)-4', $5^{\prime}, 6^{\prime}, 7^{\prime}$-四氢- $5^{\prime}, 7^{\prime}$ 桥亚甲基-吲唑-1'-基]乙酰胺 (4c): 得率为 76.7\%, 褐色 粉末状固体, m.p.143 $144{ }^{\circ} \mathrm{C} ;{ }^{1} \mathrm{H}$ NMR (DMSO- $d_{6}, 500$ MHz) $\delta: 0.71(\mathrm{~s}, 3 \mathrm{H}), 1.41(\mathrm{~s}, 3 \mathrm{H}), 1.34 \sim 1.36(\mathrm{~m}, 1 \mathrm{H})$, $2.33(\mathrm{~s}, 1 \mathrm{H}), 2.53(\mathrm{~s}, 1 \mathrm{H}), 2.70 \sim 2.72(\mathrm{~m}, 1 \mathrm{H}), 2.82 \sim 2.97$ (m, 2H), 4.70 (s, 2H), 7.60 (d, $J=4.3 \mathrm{~Hz}, 2 \mathrm{H}), 7.72$ (d, $J=$ $4.3 \mathrm{~Hz}, 2 \mathrm{H}), 7.19(\mathrm{~s}, 1 \mathrm{H}), 7.39(\mathrm{~s}, 1 \mathrm{H}) ;{ }^{13} \mathrm{C} \mathrm{NMR}$ (DMSO- $\left.d_{6}, 125 \mathrm{MHz}\right) \delta: 21.17,25.85,25.95,31.81,41.07$, 51.00, 59.63, 108.77, 119.69, 127.23, 131.33, 133.57, 144.16, 150.91, 168.47; IR (KBr) v: 3328, 3182, 2925, 2213, 1902, 1740, 1685, 1632, 1523, 1452, 1398, 1362, 1322, $828 \mathrm{~cm}^{-1}$; MS (70 eV) $\mathrm{m} / z(\%): 373\left(\mathrm{M}^{+}, 48\right), 360$ (12), 358 (13), 346 (10); ESI-HRMS calcd for $\mathrm{C}_{18} \mathrm{H}_{21} \mathrm{BrN}_{3} \mathrm{O}[\mathrm{M}+\mathrm{H}]^{+}$374.0862, found 374.0871.

2-[6', $6^{\prime}$-二甲基-3'-(4' - 甲氧基苯基)-4', $5^{\prime}, 6^{\prime}, 7^{\prime}$ - 四 氢 - $5^{\prime}, 7^{\prime}$ - 桥亚甲基-吲唑- $1^{\prime}$ - 基] 乙酰胺 (4d): 得率为 $78.7 \%$, 棕黄色粉末状固体. m.p. $147 \sim 148{ }^{\circ} \mathrm{C} ;{ }^{1} \mathrm{H}$ NMR $\left(\mathrm{DMSO}_{6} d_{6}, 500 \mathrm{MHz}\right) \delta: 0.69$ (s, 3H), $1.38(\mathrm{~s}, 3 \mathrm{H}), 1.32 \sim$ $1.34(\mathrm{~m}, 1 \mathrm{H}), 2.30(\mathrm{~s}, 1 \mathrm{H}), 2.66(\mathrm{~s}, 1 \mathrm{H}), 2.67 \sim 2.68(\mathrm{~m}$, 1H), $2.88 \sim 2.92(\mathrm{~m}, 2 \mathrm{H}), 3.77$ (s, 3H), 4.64 (s, 2H), 6.96 (d, $J=4.3 \mathrm{~Hz}, 2 \mathrm{H}), 7.68$ (d, $J=4.3 \mathrm{~Hz}, 2 \mathrm{H}), 7.15(\mathrm{~s}, 1 \mathrm{H})$, $7.30(\mathrm{~s}, 1 \mathrm{H}) ;{ }^{13} \mathrm{C}$ NMR (DMSO- $\left.d_{6}, 125 \mathrm{MHz}\right) \delta$ : 21.20 , 25.90, 26.03, 31.87, 40.59, 41.17, 50.88, 54.98, 107.84, $113.84,126.55,127.12,145.22,150.48,158.21,168.75$; IR (KBr) v: 3418, 3154, 2952, 2840, 1696, 1666, 1610, 1519, 1386,1300, 1248, 1181, 1107, $837 \mathrm{~cm}^{-1}$; MS (70 eV) $\mathrm{m} / \mathrm{z}$ (\%): 325 ( $\left.\mathrm{M}^{+}, 89\right), 310$ (17), 296 (6), 282 (47), 265 (18); ESI-HRMS calcd for $\mathrm{C}_{19} \mathrm{H}_{24} \mathrm{~N}_{3} \mathrm{O}_{2}[\mathrm{M}+\mathrm{H}]^{+} 326.1863$, found 326.1873 .

2-[6', $6^{\prime}$-二甲基- $3^{\prime}$-( $2^{\prime}$-氯苯基)-4', $5^{\prime}, 6^{\prime}, 7^{\prime}$-四氢- $-5^{\prime}, 7^{\prime}$ 桥亚甲基-吲唑- $1^{\prime}$-基]乙酰胺 (4e): 得率为 $79.3 \%$, 浅黄 色粉末状固体, m.p. 141 142 ${ }^{\circ} \mathrm{C}$; ${ }^{1} \mathrm{H}$ NMR (DMSO- $d_{6}$, $500 \mathrm{MHz}) \delta: 0.75(\mathrm{~s}, 3 \mathrm{H}), 1.42(\mathrm{~s}, 3 \mathrm{H}), 1.34 \sim 1.36(\mathrm{~m}$, $1 \mathrm{H}), 2.28(\mathrm{~s}, 1 \mathrm{H}), 2.53(\mathrm{~s}, 1 \mathrm{H}), 2.66 \sim 2.67(\mathrm{~m}, 1 \mathrm{H}), 2.82 \sim$ $2.97(\mathrm{~m}, 2 \mathrm{H}), 4.04(\mathrm{~s}, 1 \mathrm{H}), 4.70(\mathrm{~s}, 2 \mathrm{H}), 7.40$ (d, $J=4.0$ $\mathrm{Hz}, 2 \mathrm{H}), 7.53$ (d, $J=4.0 \mathrm{~Hz}, 2 \mathrm{H}), 7.20(\mathrm{~s}, 1 \mathrm{H}), 7.30$ (s, $1 \mathrm{H}$ ); ${ }^{13} \mathrm{C}$ NMR (DMSO- $\left.d_{6}, 125 \mathrm{MHz}\right) \delta: 21.25,25.04$, 25.91, 32.09, 40.99, 42.50, 50.96, 110.65, 126.79, 129.17, 129.52, 131.70, 131.90, 133.38, 145.17, 149.68, 167.76, 168.56; IR (KBr) v: 3337, 3185, 2912, 1687, 1527, 1448, 1401, 1324, 1252, $761 \mathrm{~cm}^{-1}$; MS (70 eV) $\mathrm{m} / z(\%): 329$ $\left(\mathrm{M}^{+}, 41\right), 314$ (14), 288 (20), 287 (18); ESI-HRMS calcd for $\mathrm{C}_{18} \mathrm{H}_{20} \mathrm{ClN}_{3} \mathrm{NaO}[\mathrm{M}+\mathrm{Na}]^{+}$352.1187, found 352.1199.

2-[6 $6^{\prime}, 6^{\prime}$-二甲基- $3^{\prime}$-(4' - 硝基苯基)-4', $5^{\prime}, 6^{\prime}, 7^{\prime}$ - 四氢$5^{\prime}, 7^{\prime}$-桥亚甲基-吲唑-1'-基]乙酰胺(4f): 产率 $80.3 \%$, 浅
黄色粉末状固体, m.p. 197 198 ${ }^{\circ} \mathrm{C} ;{ }^{1} \mathrm{H}$ NMR (DMSO$\left.d_{6}, 500 \mathrm{MHz}\right) \delta: 0.72(\mathrm{~s}, 3 \mathrm{H}), 1.42(\mathrm{~s}, 3 \mathrm{H}), 1.35 \sim 1.37(\mathrm{~m}$, $1 \mathrm{H}), 2.35(\mathrm{~s}, 1 \mathrm{H}), 2.53(\mathrm{~s}, 1 \mathrm{H}), 2.72 \sim 2.74(\mathrm{~m}, 1 \mathrm{H}), 2.89 \sim$ $3.34(\mathrm{~m}, 2 \mathrm{H}), 4.76(\mathrm{~s}, 2 \mathrm{H}), 8.01(\mathrm{~d}, J=4.4 \mathrm{~Hz}, 2 \mathrm{H}), 8.28$ (d, $J=4.4 \mathrm{~Hz}, 2 \mathrm{H}), 7.22(\mathrm{~s}, 1 \mathrm{H}), 7.47(\mathrm{~s}, 1 \mathrm{H}) ;{ }^{13} \mathrm{C} \mathrm{NMR}$ (DMSO- $\left.d_{6}, 125 \mathrm{MHz}\right) \delta: 21.16,25.80,26.03,31.7640 .57$, $41.00,51.21,110.28,123.92,125.86,140.82,143.30$, 145.71, 151.49, 168.25; IR (KBr) v: 3441, 3150, 3000, 2955, 1700, 1598, 1514, 1337, 1392, 1304, 854, $765 \mathrm{~cm}^{-1}$; MS (70 eV) m/z (\%): $340\left(\mathrm{M}^{+}, 22\right), 325$ (4), 315 (6), 310(8); ESI-HRMS calcd for $\mathrm{C}_{18} \mathrm{H}_{21} \mathrm{~N}_{4} \mathrm{O}_{3}[\mathrm{M}+\mathrm{H}]^{+}$ 341.1608 , found 341.1619 .

2-[6', $6^{\prime}$-二甲基-3'-(4' - 氟苯基)-4', $5^{\prime}, 6^{\prime}, 7^{\prime}$-四氢- $5^{\prime}, 7^{\prime}$ 桥亚甲基-吲唑-1'-基]乙酰胺 $(\mathbf{4 g})$ : 产率 83.7\%, 白色粉 末状固体. m.p. $165 \sim 166{ }^{\circ} \mathrm{C} ;{ }^{1} \mathrm{H}$ NMR (DMSO- $d_{6}, 500$ $\mathrm{MHz}) \delta: 0.72(\mathrm{~s}, 3 \mathrm{H}), 1.41(\mathrm{~s}, 3 \mathrm{H}), 1.34 \sim 1.39(\mathrm{~m}, 1 \mathrm{H})$, $2.69 \sim 2.73(\mathrm{~m}, 1 \mathrm{H}), 2.82 \sim 2 . .86(\mathrm{~m}, 1 \mathrm{H}), 2.93 \sim 2.97(\mathrm{~m}$, 2H), 4.69 (s, 2H), 7.24 (d, $J=8.6 \mathrm{~Hz}, 2 \mathrm{H}), 7.80$ (d, $J=8.6$ $\mathrm{Hz}, 2 \mathrm{H}), 7.18$ (s, 1H), 7.37 (s, 1H); ${ }^{13} \mathrm{C}$ NMR (DMSO- ${ }_{6}$, $125 \mathrm{MHz}) \delta: 21.18,25.86,25.90,31.82,41.10,50.94$, 59.64, 108.33, 115.22, 127.13, 130.91, 144.41, 150.78, 160.16, 162.10, 168.57; IR (KBr) v: 3329, 3180, 2926, 2840, 1687, 1635, 1526, 1460, 1364, 1323, 1153, 807 $\mathrm{cm}^{-1}$; MS (70 eV) m/z (\%): $313\left(\mathrm{M}^{+}, 42\right), 298$ (12), 270 (40), 225 (100); ESI-HRMS calcd for $\mathrm{C}_{18} \mathrm{H}_{21} \mathrm{FN}_{3} \mathrm{O}$ [M+ $\mathrm{H}]^{+} 314.1663$, found 314.1675 .

\subsection{4 晶体结构测定}

将经过乙酸乙酯和石油醚重结晶后的化合物 $\mathbf{4 d}$ 溶 于二氯甲烷和乙酸乙酯中, 室温静置使其慢慢挥发, 数 天后形成淡黄色晶体，选取尺寸为 $0.35 \mathrm{~mm} \times 0.20$ $\mathrm{mm} \times 0.20 \mathrm{~mm}$ 的晶体进行 $\mathrm{X}$ 射线衍射分析. 结果表明, 化合物晶体为正交晶系, 空间群 $P 21212$, 晶胞参数为 $a=7.5508$ (3) $\AA, b=8.1627$ (4) $\AA, c=28.3787$ (13) $\AA, \alpha=$ $90^{\circ}, \beta=90^{\circ}, \gamma=90^{\circ}, Z=4, V=1749.12(14) \AA^{3}, D_{\mathrm{c}}=1.236$ $\mathrm{g} / \mathrm{cm}^{-3}, M_{\mathrm{u}}=0.082 \mathrm{~mm}^{-1}, F(000)=696$. 数据存于英国 剑桥数据中心, CCDC 号为 1482139 .

\subsection{5 抑菌活性测定}

在 96 孔分析板上, 首先将第 2 孔到第 12 孔加入 75 $\mu \mathrm{L}$ 无菌水，再将目标产物 $\mathbf{2 a} \sim 21$ 、阳性对照品阿米卡星， 酮康唑用 DMSO 配成 $500 \mu \mathrm{g} \bullet \mathrm{mL}^{-1}$ 的溶液 $150 \mu \mathrm{L}$ 加入 到第 1 孔，将目标化合物和阳性对照品分别在 96 孔分析 板上进行二倍稀释，从第 1 到第 12 孔配成一系列的浓度 梯度 $\left(500 \sim 0.245 \mu \mathrm{g} \cdot \mathrm{mL}^{-1}\right)$, 每孔含 $75 \mu \mathrm{L}$ 溶液, 以纯的 DMSO 作为参照, 再向每个孔中加入 $75 \mu \mathrm{L}$ 预先配好的 菌悬液, 充分混匀. 最后将 96 孔分析板置于 $30{ }^{\circ} \mathrm{C}$ 培养 
箱中, 细菌培养 $24 \mathrm{~h}$, 真菌培养 $48 \mathrm{~h}$ 后观察, 以不产生 混浊的最低浓度的孔对应的浓度作为该样品对该测试 菌的最低抑菌浓度. 每个样品对每种测试菌重复三次, 结果取平均值.

\section{2 .6 杀虫活性测定}

采用浸渍法, 测定供试化合物对紫薇长斑蚜的毒力 效果. 以质量分数为 $0.1 \%$ Triton X-100 水溶液为对照, 试验时母液用质量分数为 $0.1 \%$ Triton X-100 水溶液稀 释成所需要的系列浓度梯度, 将带有紫薇长斑蚜的叶片 摘下, 用细小毛笔将紫薇叶片上过小的蚜虫剔除, 仅保 留无翅成蚜和个体较大的无翅若蚜, 每处理 $20 \sim 30$ 头 蚜虫, 重复 3 次. 然后将叶片浸于已配好的不同浓度的 药液中, 浸渍 $10 \mathrm{~s}$, 迅速取出, 用滤纸吸去多余药液后, 放置到直径为 2.5 厘米的无药指形管中, 用脱脂棉球堵 塞管口, 置于温度为 $(25 \pm 1){ }^{\circ} \mathrm{C}$, 光周期为 $16: 8 \mathrm{~h}(\mathrm{~L}$ : D)的培养箱内, $24 \mathrm{~h}$ 后检查死亡率. 检查时用毛笔轻轻 拨动虫体, 不能动即视为死亡.

辅助材料(Supporting Information) 化合物的氢谱、碳 谱、红外谱图以及高分辨质谱. 这些材料可以免费从本 刊网站(http://sioc-journal.cn/)上下载.

\section{References}

[1] Abdel-Hafez, E. S. M. N.; Abuo-Rahma, E. D. A. A.; Abdel-Aziz, M.; Radwan, M. F.; Farag, H. H. Bioorg. Med. Chem. 2009, 17, 3829.

[2] Christodoulou, M. S.; Liekens, S.; Kasiotis, K. M.; Haroutounian, S. A. Bioorg. Med. Chem. 2010, 18, 4338.

[3] Gouda, M. A.; Berghot, M. A.; Shoeib, A. I.; Khalil, A. M. Eur. J. Med. Chem. 2010, 45, 1843.

[4] Cheng, Y.; Wang, H.; Dinesh, A.; Zhou, H. C. J. Org. Chem. 2016, 36, 1 (in Chinese). (程宇, 王辉, Dinesh Addla, 周成合, 有机化学, 2016, 36, 1.)

[5] Xia, Q. C.; He, Q. Z.; Xu, D. F.; Sun, D. Z.; Peng, Z. F. Acta Chim. Sinica 2010, 68, 2414 (in Chinese).

(夏庆春, 何其庄, 许东芳, 孙大志, 彭子飞, 化学学报, 2010, 68,2414 .)
[6] Gökhan-Kelekçi, N.; Yabanoğlu, S.; Küpeli, E.; Salgın, U.; Özen, O.; Uçar, G.; Yeşilada, E.; Kendi, E.; Yeşilada, A.; Bilgin, A. A. Bioorg. Med. Chem. 2007, 15, 5775.

[7] Lv, P. C.; Li, H. Q.; Sun, J.; Zhou, Y.; Zhu, H. L. Bioorg. Med. Chem. 2010, 18, 4606.

[8] Bandgar, B. P.; Gawande, S. S.; Bodade, R. G.; Gawande N. M.; Khobragade, C. N. Bioorg. Med. Chem. 2009, 17, 8168.

[9] Farag, A. M.; Ali, K. A.; El-Debss, T. M.; Mayhoub, A. S.; Amr, A. G. E.; Abdel-Hafez, N. A.; Abdulla, M. M. Eur. J. Med. Chem. 2010, 45, 5887.

[10] Liu, T. T.; Ni, Y.; Zhong, L. K.; Huang, H. Y.; Hu, W. Q.; Xu, T. M.; Tan, C. X. Chin. J. Org. Chem. 2015, 35, 422. (in Chinese). (刘婷婷, 倪芸, 钟良坤, 黄红英, 胡伟群, 许天明, 谭成侠, 有 机化学, 2015, 35, 422.)

[11] Liu, X. H.; Bai, L. S.; Wang, S. F. Chin. J. Synth. Chem. 2006, 14, 147 (in Chinese). (刘新华，白林山，王世范，合成化学, 2006, 14, 147.)

[12] Wen, L. R.; Li, M.; Jing, S. X. Chin. J. Org. Chem. 2005, 25, 197 (in Chinese). (文丽荣，李明，景淑霞，有机化学, 2005, 25, 197.)

[13] Zhang, D. Q.; Xu, G. F.; Liu, Y. H.; Wang, D. Q.; Yang, X. L.; Yuan, D. K. J. Org. Chem. 2015, 35, 2191 (in Chinese). (张大强, 徐高飞, 刘艳红, 王道全, 杨新玲, 袁德凯, 有机化学, 2015, 35, 2191.)

[14] Liu, J.; Xie, H. P.; Song, B. A. Chin. J. Org. Chem. 2010, 30, 1347 (in Chinese). (刘杰，谢化鹏，宋宝安，有机化学，2010, 30, 1347.)

[15] Bu, Y. Q.; Shan, Z. J.; Zhou, J. Y.; Lin, X. L. Agrochemicals 2009, 48, 399 (in Chinese). (卜元卿，单正军，周军英，林小丽，农药, 2009, 48, 399.)

[16] Prakash, A.; Rao, J.; Nandagopal, V. J. Biopesticides 2008, 1, 154.

[17] Rufino, A. T.; Ribeiro, M.; Judas, F. J. Nat. Prod. 2014, 77, 264.

[18] Rodrigues, K. A. D. F.; Amorim, L. V.; Dias, C. N.; Moraes, D. F. C.; Carneiro, S. M. P. J. Ethnopharmacol. 2015, 160, 32.

[19] Kim, D. S.; Lee, H. J.; Jeon, Y. D.; Han, Y. H.; Kee, J. Y.; Kim, H. J.; Kim, S. J. Am. J. Chin. Med. 2015, 43, 731.

[20] Li, J.; Tian, X.; Gao, Y. J. RSC Adv. 2015, 5, 66947.

[21] Gao, Y.; Li, J.; Shang, S. Lett. Drug Des. Discovery 2015, 12, 241.

[22] Liu, B.; Wang, S. F. J. Nanjing For. Univ. 2010, 34, 89 (in Chinese). (刘兵, 王石发, 南京林业大学学报, 2010, 34, 89.)

[23] Wei, B. S.; Xu, X.; Yang, Y. Q.; Cao, X. Q.; Wang, S. F. J. Org. Chem. 2012, 32, 287 (in Chinese). (魏柏松, 徐徐, 杨益琴, 曹晓琴, 王石发, 有机化学, 2012, 32, 287.) 